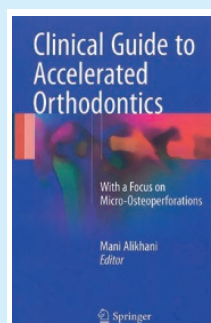

\section{CLINICAL GUIDE TO ACCELERATED ORTHODONTICS}

\author{
Mani Alikhani \\ Springer, 2017 \\ Price $\mathrm{f} 82.00$ pp. 132 \\ ISBN: 9783319433998
}

This book is intended as a reference for clinicians who are interested in incorporating techniques available to reduce the duration of orthodontic treatment and overcome other treatment limitations. The book is available as a textbook or eBook. It has an organised structure that is simple to use and allows specific topics to be located easily from the contents page at the beginning of each chapter.

The book is divided into seven chapters, each with an introduction leading to detailed discussion and sub-sections followed by a list of references. It is written by nine authors who all have links with the Consortium for Translational Orthodontic Research, a centre dedicated to translating bench and animal research into improved and innovative orthodontic therapies.

The main focus of the book is micro-osteoperforations (MOPs) and how their catabolic and anabolic effects can be utilised during orthodontic treatment. The authors present the various benefits of
MOP treatment including reduced orthodontic treatment time by accelerating tooth movement, expanding the envelope of orthodontic corrections through cortical drift and a reduction of some of the risks associated with treatment such as root resorption.

The early chapters give an overview of biological tooth movement and the current theories around its initiation before moving on to the different methods to accelerate tooth movement that have been described and their relative strengths and weaknesses. Chapters three to five give an introduction to MOPs and provide an overview of their anabolic and catabolic effects and how these may influence tooth movement. Chapters six and seven provide a step-by-step guide to performing MOP procedures and how conventional treatment planning can be modified to include MOPs in orthodontic cases and how this may benefit the patient.

The book is written in an easy to read manner and includes numerous high quality colour illustrations and photographs, which break up the text and help to reinforce the concepts being described. I particularly enjoyed the clinical cases included to demonstrate the use of MOPs in challenging cases.

I feel that this book will appeal to orthodontists who wish to learn more about methods to reduce treatment times and overcome certain treatment limitations.

J. P. R. Smyth 